\title{
Doing the Transparent State: open government data as performance indicators
}

\author{
Evelyn Ruppert
}

2015 (forthcoming). In $A$ World of Indicators: The making of governmental knowledge through quantification. R. Rottenburg, S. E. Merry, S.-J. Park and J. Mugler. Cambridge: Cambridge University Press.

\section{Introduction}

Data is the new raw material of the 21st century; it allows citizens to hold governments to account, drives improvements in public services by informing choice, and provides a feedstock for innovation and growth. ${ }^{1}$

In 2010 the Cabinet Office of the UK government introduced its Transparency Agenda (TA) as a key part of its efficiency and reform programme and as a means of taking power away from Whitehall and putting it in the hands of the public. Through the oversight of the Public Sector Transparency Board all Whitehall departments must publish key public datasets - from expenses to business plans - on their websites in specified open data standards as a means of advancing a public right to data. A number of platforms then consolidate all of the data via online and interactive access points. In addition to spurring service delivery improvements and stimulating the digital economy, the TA is said to be liberating and making government data more accessible, interesting and dynamic via websites, mobile device apps and other platforms. Along with powerful visualisation devices quantitative data about the performance of the state is being rendered accessible and legible in innovative ways. Through the TA government data is on the move and traveling to myriad sites and even sceptics see the possibilities that are opened up by this 'big data.'

As the title of this chapter suggests open government data constitute indicators of the state's performance just as indicators in general make phenomena visible so that they can be assessed, compared, and ranked. The TA ostensibly renders the state visible to the scrutiny of the governed such that the gaze is turned from the governed back onto the governors where subjects of 'dataveillance' now engage in 'sousveillance' - a 'watchful vigilance from underneath' (Dennis 2008). In a time of Wikileaks, MPs expenses and open government data, it is tempting to conclude that 'seeing like a state' (Scott 1998) is being transformed into 'seeing a state'. However, rather than considering the TA as a practice of disclosure and oversight, I argue that the transparent state is being done by a changing configuration of mediators that is generative of data publics and multiple versions of the state and the very condition that it seeks to eradicate: distrust in the workings of the state.

To develop and unpack this argument I first step back and return often to consider another kind of device that sought to make the workings of science transparent in the 17th century and constitute matters of scientific fact. Shapin (1984) writes an account of scientist Robert Boyle's experiments in pneumatics that involved constructing an elaborate air pump to demonstrate how a working 
vacuum could be created by emptying air from a cylinder. The experiments not only produced new knowledge of the behaviour of air, but established how legitimate knowledge and 'matters of fact' could be produced and evaluated by multiplying the witnessing of a sanctioned experimental practice. He writes:

Boyle proposed that matters of fact be generated by a multiplication of the witnessing experience. An experience, even of an experimental performance, that was witnessed by one man alone was not a matter of fact. If that witness could be extended to many, and in principle to all men, then the result could be constituted as a matter of fact. In this way, the matter of fact was at once an epistemological and a social category (Shapin 1984: 4834).

Shapin identifies three ways that collectivising and multiplying witnessing was achieved beyond the confines of the scientist's laboratory: demonstrations before authoritative individuals; facilitating replicability and thus the repetition of demonstrations; and virtual witnessing. All were accomplished using three inter-related technologies: the material technology required for the operation of the air-pump; the social technology of scientific rules and conventions for considering knowledge-claims; and the literary technology of detailed experimental reports that could multiply witnessing beyond the laboratory. Shapin argues that the latter were most effective for multiplying witnessing. For one, material replications of experiments rarely succeeded even though Boyle intricately communicated how the engine worked and the necessary experimental procedures (490). Thus, detailed experimental reports, and words and images, were more important as they enabled numerous readers to imagine experiments without direct witnessing or replication. A literary technology was thus central to the generation of both a scientific public and the constitution of matters of fact as a social category.

Shapin's account provides an interesting starting and reference point for thinking about how governments seek transparency about their workings and to establish matters of fact by disseminating data on the Internet and multiplying the 'virtual witnessing' of the state. Numerous governments are embracing Web 2.0 technologies to change the traditional ways of reporting on their doings such as annual reports that communicate official statistics. Each seeks to liberate data' from the confines of administrative offices and make them publicly available along with applications and tools for imagined publics to do their own analyses of 'raw data.' Transparency, democracy, accountability, engagement, collaboration, participation - these are just a few norms politicians and administrators attach to providing open government data. For some, transparency represents a new 'freedom of information' and because it mobilises interactive Web technologies constitutes Gov 2.0.

Like the technologies Shapin identified, the TA mobilises all three kinds of technologies - material, social and literary - to establish facts about the performance of the state. But it is different in three ways that I summarise here and then elaborate below. First, instead of beckoning a virtual witnessing public, the TA generates 'data publics' who are not recipients of experimental reports, information or expert generated analyses and audits but are incited to do their 
own analyses through material, social and literary (especially visual) means. This is a different from Boyle's literary technologies, which brought the public 'into the experimental scene' (511) by calling upon them to reproduce experiments in their minds. Instead of learned and credentialed authoritative men of science, the TA calls forth data publics who can do their own experiments, establish matters of fact, see the state for themselves and disseminate their results to others. Witnessing is thus turned into doing such that the literary technologies of auditor statements or government annual reports are displaced by myriad analyses conducted by imagined data publics. While disciplinary and organisational practices have legitimised the expertise of scientists such that there could be trust in their numbers (Porter 1995), trust in analyses of TA data is derived from the presumed openness and publicity of data whereby transparency is afforded moral superiority.

A second difference concerns the arrangements that constitute data publics. Like scientists data publics are configured by the sociotechnical arrangements of which they are a part. But the arrangements that make up data publics are quite distinct from the institutions, scientific rules and conventions, and disciplinary arrangements that configure the fact-making practices of scientists. Data publics are constituted by dynamic, complex and uncertain arrangements of actors mobilised and provoked by open data such as technicians, bureaucrats, software technologies, data formats, and so on or all of the material, social and literary technologies required to translate data into information. It is through a changing configuration of mediators that data publics are being produced and brought into being. For this reason I suggest these arrangements are best understood as agencements, a concept that captures how the agency and action of humans and technologies are mutually constituted.

Third, not only are data publics brought into being but it is through the relations and actions of never fixed people and things that the transparent state is being enacted or done: that is, the TA does not discover or reveal the state, but brings into being the 'real' and represented state simultaneously. But just as many data publics are generated so too is the transparent state made multiple. ${ }^{2}$ Indeed, the interactive and regularly updated data, the endless generation of new data and changing relations between myriad humans and technologies lead to multiple enactments. The performance of the state is thus never settled and the transparent state must be constantly replicated and done. Virtue is thus found in openness and in the constant generation of multiple accounts. Consequently, there can only ever be more to reveal and different accounts of the state to be generated. But this is complicated further: because the TA draws boundaries around what is to be included/excluded, displaces implicit knowledge, and reconfigures bureaucratic recording practices it is generative of distrust and leads to what Strathern (2000a) has called the 'tyranny of transparency.'

In the absence of trust and possibility of transparency, I conclude that what is needed is not more of the same but analytics that can attend to the normative and political assumptions embedded in the versions of the state that the TA elevates and makes possible. To begin I briefly outline the myriad data that make up the TA and suggest that they constitute two kinds of measurements of the workings of the state: transactional and evaluation metrics. I outline how these are different from the numerical accounts generated by audits and part of 
agencements that call forth data publics, reconfigure expertise and social knowledge and generate multiple enactments of the state.

\section{Transparency Agenda}

Notably, the TA was introduced in the wake of the 2009 MPs expenses scandal, an online data exposé led by the media which involved public exposure of MPs spending patterns on the Internet and led to more criminal convictions, resignations or decisions to stand down in a subsequent election than any other single event or controversy in recent history (Ruppert and Savage 2012). The TA needs to be understood in part as a reaction to this media exposure and as an effort to prevent such moral failures from happening again in the future (Harvey, Reeves, and Ruppert 2013 (Forthcoming)). Indeed upon election one of the first actions of the Conservative-Liberal Democrat Coalition government was the establishment of the Public Sector Transparency Board in June 2010. The Board oversees the TA including the setting of open data standards across the public sector and ensuring the opening up of data sets in machine readable formats to allow for data linking and analysis. ${ }^{3}$

The TA is made up of seven elements that extend concerns about MPs expenses to the activities, expenditures and policies of every department.

1. Who does what in Whitehall. All senior civil servants and their salaries are displayed in standard organisational charts that are dynamic and interactive. For example, we can move about the Department of Business Innovation and Skills (DBIS) and learn that the Permanent Secretary, Martin Donnelly earns an annual salary of $£ 160,000$ - 164,999.

2. Who ministers are meeting. Data is provided on all ministerial external meetings (who, purpose), hospitality (meals), gifts received (values) and overseas travel (where, how, purpose, cost). For example, in May and June 2010, we can learn that the Rt Hon Kenneth Clarke QC MP, Lord Chancellor and Secretary of State for Justice received 'hospitality' from journalists at The Times, The Daily Mail, Channel 4, and BBC. He also had 'introductory meetings' with external organisations such as the Metropolitan Police and a 'stakeholder meeting' with the Bishop of Liverpool. He travelled to Luxembourg from the 3rd to 4th June 2010 for a Justice and Home Affairs Committee Meeting via Eurostar that cost $£ 782$.

3. Government contracts in full. A 'Contracts Finder' provides access for businesses, government buyers and the public to live contract opportunities, closed tender documentation, contract awards and contract documents for all procurements above $£ 10,000$. For example, in June 2012 English Heritage awarded Swift Fire \& Security a $£ 11,974$ contract to replace two security bollards at Kenwood House, London.

4. How your money is spent. Monthly expenditures of all central government spending over $€ 25,000$ broken down by department are provided. For example, on 12th May 2010, the Ministry of Justice paid $£ 437,916.63$ to Atos Origin IT Services UK Ltd. for IT and Telecommunications. This is only one of 64 itemised expenditures made on that day.

5. Business plans. Departments must monitor and provide monthly reports on their progress in meeting priorities set out in their business plans, which 
implement the governments 'structural reform plans.' For example, in February 2011 the DBIS completed the development of proposals to introduce loans for FE students and reported that work was on-going to reform quality-related research funding.

6. All other government data. Every department must have an information strategy explaining how it will share data and promote transparency. In addition to the above, input indicators are required on how the department will measure the efficiency of its policies and expenditures, and impact indicators setting out measurements to evaluate policy outcomes. For example, the DBIS adopted the following input indicators: funding per student in higher education and total number of researchers in UK universities. The impact indicators include the qualification levels of the working age population in England.

In addition to these datasets, departments are also required to make available data that are used in their published reports and collected via various means such as surveys. For example, the DBIS provides spreadsheets on surveys of English businesses, and on building materials and trade union membership.

7. MPs Expenses. A final component follows from the MPs expenses scandal mentioned above. Expenses are now compiled and released through the Independent Parliamentary Standards Authority (IPSA). ${ }^{4}$ The IPSA transparency webpage provides data on annual expense totals for all MPs, itemisations of expenses for each MP (e.g., travel), and details for each transaction (e.g., origin-destination).

All of the above data can be accessed through several portals and most of it via machine readable formats: the IPSA website just noted; the PMO's Number 10 Transparency homepage, the transparency pages of departments and through the open government data platform that is overseen by the Transparency Board, which as of Sept 2012 boasts some 8667 datasets. ${ }^{5}$

What can we make of this myriad data? First, it can be understood as constituting two kinds of metrics or quantitative measurements to evaluate the performance of the state. One is a transactional metric and consists of data collected as a 'by-product' of service delivery (e.g., departmental purchases and contracts); 6 the activities of Ministers (meetings, gifts); the remuneration of senior bureaucrats; and, direct programme expenditures (e.g. total spend on museums). Most of this data is devoid of reference to the substance of government work and instead are simply quantifications of transactions. For example, little is revealed about the content of meetings, the reasons and uses of a purchase or a particular salary and so on. Instead the data are detailed and granular quantitative registers of the heterogeneous doings of the state over time.

The second kind is an evaluation metric. This includes data on progress towards achieving business plans which can be tracked in 'real-time'; socio-demographic data on the performance of the population (e.g., health statistics); and, input/impact indicators for policy decision-making and service delivery assessment. Instead of a language of targets (as established under the New Labour government) indicators are adopted to replace top-down systems and 
micromanagement. Rather than centrally defined targets, the assumption is that departments are best positioned to define and evaluate their performance according to indicators they establish. In general, evaluation metrics can be understood as measurements of the consequences of government transactions (meetings, expenses, programme costs) that implement policies (e.g., on the economy or health of the population).

Taken together, the TA consists of metrics of the everyday granular transactions of the state and their outcomes. It is because they are recordings of the states doings and units for measuring, monitoring and evaluating its performance that I refer to the data as metrics. Furthermore, as I will describe later, rather than any one piece of data, it is through comparisons, rankings and the identification of patterns in the data that performance is revealed and assessed. In some ways, these metrics are similar to the measurements that constitute an audit, which Power (1999) argues exploded in the late 1980s and early 1990s and was in part about control and organisational transparency. Generally, auditing involves 'checking up' on public bodies through systems of verification. In an audit culture, indicators and benchmarks place responsibility on the performer, not the checker: 'Responsibility for compliance shifts to the monitored organisation, corporation, or country itself, which must not only seek to comply but also monitor and report the success of its efforts' (Engle Merry 2011: S88). While Power's focus is on financial auditing, many practices engage in similar procedures of checking and reporting such as those of university research assessment exercises (Strathern 2000b). Power argues that what exploded was less a standardised technique - there are numerous practices that are described as auditing - and instead an 'idea' about 'ritualised practices of verification' (14). Rather than a weakness, he deems that this is what makes auditing so powerful as it refers to a 'style' of indirect regulation or control that can be configured in a variety of ways.

If Boyle required a witnessing public that could read texts and visualisations to verify and establish matters of fact, so too does the auditor when publishing his/her accounts. And both also involve responsibilising and forms of indirect control. But there are important differences between the audit and the metric. For one, with the TA ritualised practices of verification move from the offices of auditors and accountants to the online platforms of publics. And second, they move verification from paper ledgers, auditor statements and reports to detailed digital recordings that imagined publics can order, reorder and experiment with. These qualities constitute a ritualised practice that calls for different kinds of publics to verify matters of fact. While Boyle's experiments required publics that could read and understand experimental reports, the TA requires publics that can analyse and do things with data.

\section{Producing Data Publics}

But now statistics have become democratised, no longer the preserve of the few but of everyone who has a spreadsheet package on their laptop, desktop or even their mobile and tablet. Anyone can take on a fearsome set of data now and wrangle it into shape. Of course, they may not be right, but now you can easily find 
someone to help you. We are not wandering alone any more (Rogers 2011).

Transparency devices are staged 'as if' everyone can easily participate and witness the inner workings of the state. Instead, as many studies have argued, these devices are implicated in producing digital inequality and a 'digital divide' along lines of class, gender, race, and ethnicity. ${ }^{7}$ Of course, the same could be said of other techniques through which states deliver data and information to publics, such as audits and annual reports, where differences in access and literacy also lead to divisions and inequalities. The solutions put forward to reduce such 'digital divides' often include education, training and the delivery of publicly accessible technology. But such assessments and remedies treat technology as neutral and subjects as pre-formed just as the quote above highlights a common interpretation that data, devices and publics are ontologically separate, independent and pre-formed rather than performed. As Halford and Savage (2010) suggest, these assessments separate 'technology, on the one hand, from social process, on the other' (937). Rather than assuming that 'pre-formed social groups "use" (or don't use) technologies', they identify a 'more complex process of mutual interaction and stabilisation' where digital technologies are involved in constituting subjects in diverse and pervasive ways (952). For example, Wilson (2011) has shown how geocoding subjects with particular urban imaginations and cartographic visions are formed through technologies of citizen engagement such as handheld devices.

In this view we can think about the publics and technologies making up the TA as mutually constituting, as ontologically relational rather than independent categories that come into play with each other (Halford and Savage 2010). As the quote above suggests, open government data requires particular kinds of publics who will not only look at data but analyse it alongside and with others. In many ways it advances what eGovernment policies and projects have sought to produce: publics that can interact with governments through the use of information and communication technologies (ICTs) (Helbig, Gil-Garcia, and Ferro 2009). With eGovernment interactive web-based platforms or Gov 2.0 publics are invited to manage their services, access information and communicate with various state agencies.

With the TA a new dimension is added that engages publics not as customers, service recipients, information gatherers or simple witnesses but as data analysts and interpreters enrolled in a citizen science of government. While most of the data released as part of the TA was previously available, much of it was buried in various on-line or paper files, and some would have required freedom of information requests in order to access. TA liberates and enables this data to travel. Furthermore, unlike paper based methods or processes under freedom of information legislation, data is not simply to be seen but to be worked on by publics. Of course, prior to the launching of the TA, many organisations and individuals called for greater government accountability and transparency, made the release of open data a political issue and also worked with data that was already accessible. ${ }^{8}$ Many also started digitising government data and releasing it on the Internet as in the Daily Telegraph's exposé on MPs expenses (Ruppert and Savage 2012). As such, we could say publics were in formation and 'coming into being' to take care of an issue that the government was not addressing 
(Marres 2005). However, by taking up the issue, the TA actively configures the subjectivities and agencies of these data publics through particular sociotechnical arrangements of humans and technologies. They are arrangements that can best be understood as agencements, a term that emphasises that the agency and action of technological and human actors are mutually constituted. ${ }^{9}$

This understanding is implicit in Shapin's account of the witnessing public when he notes that literary technologies worked to produce a witnessing public that could read reports and expert accounts of experiments. Though establishing matters of fact involved a complex arrangement of three mutually dependent technologies only the literary was 'in the hands' so to speak of the witnessing public. Access to the material technologies needed to conduct experiments was limited and the ability to replicate experiments difficult and often leading to failure and the social technology of performing experiments before eyewitnesses was confined to select 'ingenious' and credentialed men. With the TA, multiple data publics are mobilised through relations to and engagement with all three technologies which make up the TA agencements: material technologies such as computer infrastructures, websites, apps and data; social technologies including rules, data formats, software protocols; and literary technologies such as visualisations, maps, photos, matrices and profiles. Yet, their relations to each of these is mediated: while wresting facts from experts and putting them in the hands of data publics, specific kinds of 'technical capital' - skills, resources, and knowledge - are required to design and mobilise the necessary material, social and literary technologies. While the imagined public of the TA becomes multiple data publics who are active in the establishment of matters of fact, new mediators and gatekeepers are active in directing their capacities and competencies to 'work with data' either individually or collectively (Ruppert and Savage 2012).

\section{Reconfiguration of expertise}

For Prime Minister David Cameron, putting information that was previously held by a few into the hands of all will give rise to 'real people power' and a 'postbureaucratic age' where government officials no longer are the keepers, arbiters, and interpreters of data (Cameron 2009). Power over data will ostensibly reside with an imagined public. Yet most people lack the tools or expertise to make sense of the terabytes of data being released. Like other data sources such as those generated by social media, commercial, academic and scientific practices, open government generates 'big data' that can only be managed and interpreted with analytic and computational software. Tools for making sense of the data are needed because performance trends may be hidden in the details and only detectable in patterns. Thus software developers are incited to develop apps and user-friendly interfaces for different publics to 'exploit' and interrogate the data. Organisations, volunteers and software developers have been incited to produce websites and applications that arrange and analyse the data or provide simple visualisation tools. As of Sept 2012, about 230 tools and apps were promoted on the data.gov.uk platform and the following are some examples. The website 'whoislobbying' was developed because the TA provides 'too much data.' It categorises, ranks and then provides a simple visualisation of the relative importance of types of meetings (e.g., introductory) and types of organisations attending meetings (e.g., industry). Others provide interactive tools on websites 
to visualise data such as 'Where does my money go', which 'aims to promote transparency and citizen engagement through the analysis and visualisation of information about UK public spending. ${ }^{10}$ The site has a tool that enables users to select their income and then use a slider to produce alternative visualisations of how their tax is being spent in different areas (e.g. health, education). Similar tools have also been developed for mobile devices such as the 'Numberhood' app. It compiles data on key indicators, with data charts and descriptions, enabling users to examine patterns and trends on how their local area is performing on issues such as unemployment and crime. ${ }^{11}$ The 'Commons Performance Cockpit' app produces metrics on the performance of the House of Commons and individual MPs using data from the IPSA, Parliament.uk and data.gov.uk. ${ }^{12}$ The OpenlyLocal site provides access to local council data 'without having to poke around through dense, difficult-to-navigate websites.' ${ }^{13}$ An open data 'scoreboard' identifies all local authorities that provide open data and a council dashboard compares expenditures and lists the biggest business and charity suppliers.

Yet another provider of analytic tools is the news media. ${ }^{14}$ Government data sites are heralded as an opportunity to revitalise journalism through the analysis of open government data (Filloux 2009). Instead of relying on government or academic experts and reporting on their analyses, 'data journalists' do their own research and provide in-depth analyses of immigration, crime, and finance data. In some cases they also provide interactive tools for publics to visualise, investigate and download data. ${ }^{15}$ There are numerous other examples of apps and interfaces but one point I want to highlight is that data is organised into visualisations of the performance of the state in the form of rankings, dashboards, scoreboards, patterns and trends.

With the TA then, the production of data publics is mediated by software developers, journalists, think tanks, lobbyists, watchdog organisations, data visualisers, and bloggers who are the predominant experts rather than auditors, policy analysts, academics and statisticians. ${ }^{16}$ But importantly and contrary to democratising assumptions of open government data, these mediators are reconfiguring expertise and social knowledge. It is a reconfiguration that is also connected to the purported economic value of open data, which is intended to feed the digital economy through the stimulation of new analytics and applications. Big government data is not only a source of knowledge about the state but also worth billions of pounds. To that end, in late 2011 the UK government announced the formation of an Open Data Institute (ODI) to be based in Shoreditch, London and form part of what is called the 'Silicon Roundabout'. ${ }^{17}$ Its mandate is to demonstrate the commercial value of public data and to nurture innovative, data-driven businesses using open data.

Of course, the influence of IT professions in the work of governing is not confined to open data. Horrocks (2009) argues that the eGovernment agenda has increased the power and influence IT experts and consultants who act as powerful agents. They both shape and control e-government policy and call for more 'experts' thus reinforcing a growing 'consultocracy'. Through a number of government policies the role and influence of IT consultants and suppliers has increased while the capacity of the public sector to control and manage major policy areas has declined in a wide range of domains from health care to security 
(Dunleavy et al. 2006). Open government data extends this policy influence and its democratic consequences to practices of knowledge production and how and what the public knows about government. But such influence needs to be understood in relation to the various other actors and technologies that are part of the TA agencements and the digital divisions of labour that make them up. For example, 'information infomediaries' are a faction of computer scientists and engineers who operate between data producers and users and undertake major computational tasks such as aggregating, standardising, packaging, securing and processing data. ${ }^{18}$ And code, algorithms, and protocols are made up of complex sets of rules and relations written into hardware and software (Cheney-Lippold 2011). There is also incrementalism in the design of computational software such that new designs often depend on bits of pre-existing code accumulated over time (Goffey 2012). Thus, influence is much more complexly distributed and open government data is part of an emerging technoscience that is being made not simply by consultants but through practical efforts and relations between digital and other technologies, consultants, engineers, statisticians, computer scientists, technology firms and so on. Or, as I have been arguing, of multiple and complex agencements.

This includes the visualisations noted previously, which are a fundamental point of mediation. Like Boyle's literary technology where images assisted the reader, visualisation tools are more powerful than narratives and data tables. In the experimental report, visual representations could convey more detail, imitate reality and give 'the viewer a vivid impression of the experimental scene' (492). Such visual technologies have been historically deployed to ostensibly minimise the mediation of the scientist and thus constitute objectivity (Daston and Galison 2007). However, while held up to have epistemic virtue, visual technologies always mediate objectivity. As Galloway (2011) argues in the case of the digital, visualisations are foremost depictions of principles and codes of production and only secondarily of raw data. But it is perhaps when visualisation tools are in the hands of publics that such codes become ever more blackboxed. Rather than visualising an experimental scene, apps enable publics to see and analyse data right before their very eyes as well as interact with, adjust, manipulate and experience the data. It is an experience of data mediated by technologies and screens that Knorr Cetina and Bruegger (2002) have called post-social in that subjects relate to screens not only as 'doers' but as 'experiencing, feeling, reflexive and remembering beings, as bearers of the sort of experiences we tend to reserve for the sphere of intersubjective relationships' (p. 163). They show how screens bring dispersed phenomena such as the invisible market close to participants and render it interactionally present. In relation to the TA, the screen materialises the performance of the dispersed state and constitutes a post-social relation between the state and its publics. Thus it is through screens that publics are not only produced but also that the state is made interactionally present and experienced.

But it is an experience that is generative of a particular kind of subjectivity much in the way that Hayles (2009) has written about the reconfiguration of human subjectivity by information-intensive environments such as context aware technologies. Human awareness and cognition are part of what she calls the 'cognisphere' made up of an assemblage of flows and agencies between people, 
animals and machines (48). The assemblage is part of how we live but of which we are often unaware (Hayles 2006). In a similar vein, Nigel Thrift (2004) has called this the technological unconscious of living in a world of performative infrastructures animated by intelligent technological environments that are 'producing a new sense of how the world shows up' (177). Because of the ubiquity of data, Kang and Cuff (2005) also suggest that subjects develop a 'datasense' about people, places and things that draws on myriad information and data not previously accessible. In this way TA agencements can be understood as producing data publics with a sense of the state's performance, a kind of subjectivity that is perhaps less analytic and more experiential. It calls forth data publics that can play with rankings, dashboards, cockpits, scoreboards, and other visualisations and from this acquire a sense about how the state is performing. On the basis of patterns and correlations, rather than numbers or narrative, publics can 'sense' things are getting worse/better or are suspicious/normal (Harvey, Reeves, and Ruppert 2013 (Forthcoming)). But it is a sense that is not fixed or stable; just as many data publics are generated so too is the transparent state made multiple.

\section{Enacting the Transparent State}

For Boyle, literary technologies relied to a large degree on visualisations of how things were 'really done' and what they 'really were.' This in part depended on experimental reports that stated things confidently as matters of fact and thus showing 'they were not of one's own making; they were, in the empiricist model, discovered rather than invented' (496). In this regard, Boyle's air pump worked to achieve the appearance of matters of fact much like other scientific devices have worked to render knowledge objective (Porter 1995). TA similarly positions numbers, spreadsheets and visualisation software as neutral technologies that enable the production of 'objective' accounts of 'facts' without the intervention of experts. ${ }^{19}$

However, as in the case of Boyle's air pump and other scientific devices, the TA does not simply represent or make the state transparent. As I have intimated in the previous section, it is made up of agencements of mutually constituting actors and technologies that enact the transparent state. That is, rather than producing a perspective or revealing something that is 'out there' that needs to be exposed, the reality and representation of the transparent state are simultaneously being done or enacted by the TA. ${ }^{20}$ It is being done through the multiple and relational practices of myriad people and things, from the practices, technologies and relations between bureaucrats, policy analysts, politicians, computer technicians, administrators and so on to data publics. ${ }^{21}$ This understanding of the performativity of practices and the enactment of phenomena has been well developed in the social sciences. For example, Power (1999: 9) notes that audits are not simply descriptive 'but performative, projecting and enacting ideals', and once routinised in bureaucracies or written into law can be 'increasingly real and fateful' (Espeland and Stevens 1998; Porter 1995) and intervene in the 'happening of the real' (Verran 2012). There are many other examples and though the uses vary, overall they suggest that representations and the realities represented are enacted together in knowledge practices (Law 2011). Furthermore, such practices are changing as they are 
becoming increasingly digital, which has consequences for how phenomena are being enacted. ${ }^{22}$

However, enacting the transparent state is a process rather than an event. While gathering together particular elements and appearing relatively contained and coherent, the arrangements that make up the TA are not fixed. Relations are constantly changing as new data, applications, actors and relations are updated and added. The transparent state can thus be thought of as a moving target that requires ongoing monitoring because data is never finished as the following quote attests:

'[The Department of Education] will never be able to say that [all the data has been released and in every format possible], and nor will anyone else in the government. We accept that the role of government as a provider of information will never be finished, and it will never be perfect. No department will be able to guess every single need that people will have for the information it owns' (Cabinet Office Open Data Team 2012).

The state is thus never finally revealed and equally, like many digital knowledge practices, more actors are successfully enacting and often transmitting competing and innovative digital representations. The transparent state is thus being done by a style of repetition that Thrift (2004) has argued is 'more controlled and also more open-ended, a new kind of roving empiricism which continually ties up and undoes itself (186). While such multiplicity could be construed as positive, it is also generative of other effects. Strathern (2000a) for instance argues that the accumulation of multiple accounts is based on the assumption that 'what is invisible is what is simply not yet made visible' and thus there is always more to be revealed and 'further realities to uncover' (312; italics in original). Furthermore, making some things explicit only displaces rather than erases what is implicit, all of the inner workings of government such as the values, interactions and experiential knowledge of experts. The TA displaces such implicit values, assumptions and working practices that have a potential to be data but inevitably cannot and do not make it into being data - just as much of social life escapes methods of knowing and is resistant to be being gathered together (Law and Urry 2004). Yet experiential and implicit knowledge is crucial to the operating of expertise but can only work if there is trust in practitioners (Strathern 2000a: 313). The matter then is not that everything could or should be made explicit but rather attending to the distrust that mobilised transparency in the first instance.

But the explicit does not escape distrust as it involves drawing boundaries around what is to be included and thus always involves excluding. Making things explicit involves an economy of open government data where some data is excluded because of privacy and disclosure concerns, or only released to approved researchers or only provided on a cost recovery and bespoke basis (United Kingdom Statistics Authority 2012). It also has a further effect: the doing of transparency also reconfigures practices of recording and counting. Civil servants have claimed that in relation to freedom of information legislation less information is being recorded and internal communications have become less detailed and informative (Wintour 2012). Thus the very doing of transparency 
influences and configures data recording and counting practices. Together these effects tend to exacerbate the very condition that devices such as the TA seeks to eradicate - an absence of trust. For example, Freedom of Information in Scotland requests in Scotland have had the paradoxical effect of fostering ever greater suspicion that government 'really' has something to hide (John 2010). Taken together, the accumulation of endless accounts, the drawing of boundaries, the displacement of the implicit, the reconfiguring of recording practices and the paradoxical relation to trust constitute what Strathern calls the 'tyranny of transparency.' But it is perhaps a tyranny that can only be sensed through experiencing flat visualisations generated by devices that smooth out and banish all of these constituting elements from the accounts of what the TA is and does.

Transparency is thus both done and an impossibility. It is done by elevating and naturalising particular versions of the state; it is impossible because it inevitably blackboxes its own making and closes off alternatives. Yet it continues to be held up as a virtue despite voices of mistrust and criticism and claims that it has 'become an increasingly de-politicised technical process, where public participation has inconsequential political effects' (Karounos and Lampsa 2011). However, as I will argue in the final section, political effects need to be more broadly understood.

\section{Conclusion}

While transparency has been politically and morally juxtaposed against secrecy, there is a symbiotic relation between the two, for example, when decisions are made about what to disclose or when transparent data gets buried under volumes of data thus rendering it opaque (Birchall 2011). A politics of transparency must thus be understood in relation to a politics of secrecy, a relation that is also suggested in Strathern's formulation of transparency. An answer thus does not reside in extending the boundaries of the explicit as this will not lead to greater transparency or trust in the state. Indeed, such a strategy would only legitimise the technology of power through which the state has constituted transparency and through which it seeks redemption. Indeed, calls for more disclosure of the same reinforces the virtue and moral rightness of the TA and its authority and effectivity (Harvey, Reeves, and Ruppert 2013 (Forthcoming)). Rather, a politics of transparency needs to include contestation of the knowledge practices and the kinds of realities that are both promoted and eclipsed by the TA. If the transparent state is enacted rather than revealed then what versions does the TA make possible and what versions does it close off? And how might we evaluate the relative merits of alternatives? We could start by investigating the normative and political assumptions embedded in what the TA deems relevant and important indicators of the state's openness and performance. For one, as I have argued, the TA elevates metrics that track the granular transactions of the state. Thus the transparent state is enacted in relation to what it does in much the same way that businesses and governments use transactions to identify, categorise and evaluate customers and citizens. Supermarkets track, accumulate and monitor detailed transactional data on purchases and border management agencies compile and analyse digital traces on movements to identify and categorise people on the basis of what they do. Does the TA then turn the granular movements and doings of government into indicators of its performance and the object of politics? If so, then what forms of 
politics are being displaced? These are the kinds of questions that the TA raises and which I suggest call for a different analytics of what open government data is and does.

\section{Acknowledgments}

I would like to thank participants for their comments to an earlier version of this chapter presented at the workshop, A World of Indicators, Max Planck Institute for Social Anthropology, 13-15 October 2011.

\section{Notes}

1 The quote is from the introduction to the joint ESRC and Google research call on 'Google Data Analytics Social Science Research' announced on 20 August 2012.

${ }^{2}$ In her book The Body Multiple (2002), Mol decentres the ontology of a disease - lower-limb atherosclerosis - by demonstrating how it is multiply enacted through myriad situated practices.

3 Through their deliberations the data and information requirements of the TA have been specified and set out on the Cabinet Office website and the main open data government platform, data.gov.uk. To enable linked data, items in different data sources (e.g., a city) are given addresses on the web (URIs or uniform resource identifiers), and data is published about them in machine-readable formats. Members of the Board include Tim Berners Lee (attributed with 'inventing' the World Wide Web), Professor Nigel Shadbolt (University of Southampton) and Tom Steinberg, founder of mySociety, which hosts TheyWorkForYou.com, an organisation of volunteers that monitors elected MPs (speeches, attendance, ministerial statements, etc.), and their unelected Peers, and comment on what goes on in Parliament.

${ }^{4}$ http://parliamentarystandards.org.uk/

${ }^{5}$ http://transparency.number10.gov.uk; for an example of department data see http://www.bis.gov.uk/transparency/; the open government data platform can be found at http://data.gov.uk/.

${ }^{6}$ Minister Francis Maude has referred to this data as the 'by-products' of public services (Cabinet Office 2011: 3).

${ }^{7}$ See discussion of these studies and critique of the 'digital divide' in (Halford and Savage 2010).

8 There have been many active organisations in the UK such as mySociety, a non-profit company that builds websites that provide tools and access to data so that people can make better use of the internet for civic issues.

${ }^{9}$ The term is used instead of assemblage, which has a more passive connotation and for this reason has also been taken up by others, e.g., (Hardie and MacKenzie 2007; McFall 2009; Ruppert 2011).

10 The website is produced by the Open Knowledge Foundation, a not-for-profit organisation with the aim of opening up access to information and its re-use; see

http://wheredoesmymoneygo.org/about/.

11 Designed by Oxford Consultants for Social Inclusion (OCSI) who seek to 'develop and interpret the evidence base to help the public sector and other organisations deliver better services' (see http://www.numberhood.net/about/).

12 http://data.gov.uk/apps/commons-performance-cockpit. The site was designed by KeyBusinessInsight.

13 http://openlylocal.com/info/about_us. OpenlyLocal is produced by Chris Taggart, founder of CountCulture with the aim of 'making local public data open and accessible.'

${ }^{14}$ See for example, 'data journalist' Simon Rogers' Data blog page on The Guardian website: http://www.guardian.co.uk/news/datablog. 
${ }^{15}$ See for example an interactive visualisation of crime in the US: http://www.guardian.co.uk/news/datablog/interactive/2011/sep/30/crime-map-us-data.

16 One 2010 survey (Davies 2010) identified developers as overwhelmingly male (6-to-1 in survey results), and generally split between micro-enterprise and SME business in the private sector, local and national public sector institutions, and academic institutions, with a very limited representation of voluntary sector workers.

17 It is led by Transparency Board members Tim Berners-Lee and Nigel Shadbolt and to be opened in October 2012. As part of the Governments 'East London Tech City' initiative the area around the Old Street roundabout extending to Shoreditch and Hoxton is becoming a hub for media agencies and digital technology companies such as Google's 'Campus London'.

${ }^{18}$ This is a term coined by Cisco Systems to capture 'heavy lifting' data processing services provided by centralised companies for the information technology industry and forms part of what could be understood as a 'data ecosystem' (Harris 2012).

19 This is the language of the Transparency Agenda; see for example:

http://data.gov.uk/blog/new-public-sector-transparency-board-and-public-data-transparencyprinciples.

${ }^{20}$ The argument follows approaches in science and technology, material semiotics or post-ANT (e.g., (Hacking 1999; Law 2008; Mol 2002) that contend the real and represented are enacted simultaneously.

${ }^{21}$ Here I am drawing on Mol's (2002) study of ontology in medical practice where she argues that 'perspectivalism' assumes a unified object is viewed differently by different parties. Instead, an object is a 'precarious accomplishment,' which needs to be studied rather than assumed, not a singular entity but a multiplicity and outcome of multiple practices.

22 For example, recent work that has extended performativity to the digital include (Lash 2007; MacKenzie 2005; Marres 2010; Ruppert 2011; Wajcman 2007). These arguments are more generally elaborated in (Law, Ruppert, and Savage 2010). 


\section{References}

Birchall, C., 'Introduction to "Secrecy and Transparency": The Politics of Opacity and Openness', Theory, Culture \& Society, 28 (7-8) (2011), 7-25.

Cabinet Office, Making Open Data Real: A Public Consultation: HM Government, 2011. Cabinet Office Open Data Team, 'Open Data Initiative Goes Back to School', Transparency Hub, 2 February, 2012.

Cameron, D., 'A New Politics: The Post-Bureaucratic Age', The Guardian, 25 May, 2009.

Cheney-Lippold, J., 'A Newalgorithmic Identity: Soft Biopolitics and the Modulation of Control', Theory, Culture \& Society, 28 (6) (2011), 164-181.

Daston, L., and P. Galison, Objectivity, Cambridge, Mass. : Zone Books, 2007.

Davies, T., 'Open Data, Democracy and Public Sector Reform. A Look at Open Government Data Use from Data.Gov.Uk', Masters thesis, Social Science of the Internet, Oxford, 2010.

Dennis, K., 'Keeping a Close Watch - the Rise of Self-Surveillance and the Threat of Digital Exposure', Sociological Review, 56 (3) (2008), 347-357.

Dunleavy, P., H. Margetts, S. Bastow, and J. Tinkler, Digital Era Governance: IT Corporations, the State, and E-Government, Oxford: Oxford University Press, 2006.

Engle Merry, S., 'Measuring the World: Indicators, Human Rights, and Global Governance', Current Anthropology, 52 (S3) (2011), S83-S95.

Espeland, W. N., and M. L. Stevens, 'Commensuration as a Social Process', Annual Review of Sociology 24 (1998), 313-343.

Filloux, F., 'Can Data Revitalise Journalism?', Monday Note: Media, Tech \& Business Models, 2009.

Galloway, A., 'Are Some Things Unrepresentable?', Theory, Culture \& Society, 28 (7-8) (2011), 85-102.

Goffey, A., Breaking the Laws of Code: Programming as Socio-Technical Praxis: Paper presented at the CRESC Annual Conference, Manchester, 6 September, 2012.

Hacking, I., The Social Construction of What?, Cambridge, Massachusetts: Harvard University Press, 1999.

Halford, S., and M. Savage, 'Reconceptualizing Digital Social Inequality', Information, Communication and Society, 13 (7) (2010), 937-55.

Hardie, I., and D. MacKenzie, 'Assembling an Economic Actor: The Agencement of a Hedge Fund', The Sociological Review, 55 (1) (2007), 57-80.

Harris, D., 'How Cisco Wants to Make Big Data a Community Affair', GIGAOM, 2012.

Harvey, P., M. Reeves, and E. Ruppert, 'Anticipating Failure: Transparency Devices and Their Effects', Journal of Cultural Economy, (2013 (Forthcoming)).

Hayles, N. K., 'Unfinished Work: From Cyborg to the Cognisphere', Theory, Culture \& Society, 23 (7-8) (2006), 159-66.

Hayles, N. K., 'Rfid: Human Agency and Meaning in Information-Intensive Environments', Theory, Culture \& Society, 26 (2-3) (2009), 47-72.

Helbig, N., J. R. Gil-Garcia, and E. Ferro, 'Understanding the Complexity of Electronic Government: Implications from the Digital Divide Literature', Government Information Quarterly, 26 (1) (2009), 89-97.

Horrocks, I., '"Experts" and E-Government: Power, Influence and the Capture of a Policy Domain in the Uk', Information, Communication \& Society, 12 (1) (2009), 110-127.

Kang, J., and D. Cuff, 'Pervasie Computing: Embedding the Public Sphere', Washington and Lee Law Review, 62 (1) (2005), 93-146. 
Karounos, T., and P. Lampsa, 'Introduction: Contested Transparencies Special Issue', Republic: re-imagining democracy, (2011).

Knorr Cetina, K., and U. Bruegger, 'Traders' Engagement with Markets: A Postsocial Relationship', Theory, Culture \& Society, 19 (5/6) (2002), 161-185.

Lash, S., 'Power after Hegemony: Cultural Studies in Mutation', Theory, Culture \& Society 24 (3) (2007), 55-78.

Law, J., 'On Sociology and STS', Sociological Review, 56 (4) (2008), 623-649.

- - - 'Collateral Realities', in F. Domínguez Rubio and P. Baert, (ed), The Politics of Knowledge, London: Routledge, 2011, 156-178.

Law, J., E. Ruppert, and M. Savage, Digital Devices: Nine Theses: CRESC Working Paper Series, 2010.

Law, J., and J. Urry, 'Enacting the Social', Economy and Society, 33 (3) (2004), 390-410.

MacKenzie, A., 'The Performativity of the Code: Software and Cultures of Circulation', Theory, Culture and Society, 22 (1) (2005), 71-92.

Marres, N., 'Issues Spark a Public into Being: A Key but Often Forgotten Point of the Lippmann-Dewey Debate', in B. Latour, (ed), Making Things Public: Atmospheres of Democracy, Cambridge, MA: MIT Press, 2005, 208-17.

- _ - 'Frontstaging Non-Humans: The Politics of "Green" Things and the Constraint of Publicity', in B. Braun and S. Whatmore, (ed), Political Matter: Technoscience, Democracy and Public Life, Minneapolis: University of Minnesota Press, 2010.

McFall, L., 'The Agencement of Industrial Branch Life Assurance', Journal of Cultural Economy, 2 (1-2) (2009), 49-65.

Mol, A., The Body Multiple: Ontology in Medical Practice, Durham, NC: Duke University Press, 2002.

Porter, T., Trust in Numbers: The Pursuit of Objectivity in Science and Public Life, Princeton, NJ: Princeton University Press, 1995.

Power, M., The Audit Society: Rituals of Verification, Oxford: OUP Oxford, 1999.

Rogers, S., Data Journalism at the Guardian: What Is It and How Do We Do It?: The Guardian, 2011.

Ruppert, E., 'Population Objects: Interpassive Subjects', Sociology, 45 (2) (2011), 218 233.

Ruppert, E., and M. Savage, 'Transactional Politics', in L. Adkins and C. Lury, (ed), Measure and Value: Sociological Review Monograph Series, London: WileyBlackwell, 2012.

Scott, J. C., Seeing Like a State: How Certain Schemes to Improve the Human Condition Have Failed, New Haven and London: Yale University Press, 1998.

Shapin, S., 'Pump and Circumstance: Robert Boyle's Literary Technology', Social Studies of Science, 14 (4) (1984), 481-520.

Strathern, M., 'The Tyranny of Transparency ', British Educational Research Journal 26 (3) (2000a), 309-21.

- - , ed. Audit Cultures: Anthropological Studies in Accountability, Ethics and the Academy, London and New York: Routledge, 2000b.

Thrift, N., 'Remembering the Technological Unconscious by Foregrounding Knowledges of Position', Environment and Planning D: Society and Space, 22 (1) (2004), 175190.

United Kingdom Statistics Authority, Monitoring Review: Public Availability of Official Statistics - Three ONS Datasets, London: UKSA, 2012.

Verran, H., 'Number', in C. Lury and N. Wakeford, (ed), Inventive Methods: The Happening of the Social, London: Routledge, 2012. 
Wajcman, J., 'From Women and Technology to Gendered Technoscience', Information, Communication and Society, 10 (3) (2007), 287-298.

Wilson, M. W., '"Training the Eye": Formation of the Geocoding Subject', Social \& Cultural Geography, 12 (4) (2011), 357-376.

Wintour, P., 'Freedom of Information Act Has Not Improved Government, Says MoJ', The Guardian, 13 February, 2012. 